\title{
Observed frequency of fetal trisomy between 16 and 24 gestational weeks in pregnant women older than 34 years at delivery
}

Shin Ok Jeong', You Jung Han', Si Won Lee', Dong Wook Kwak', Jin Hoon Chung', Hyun Kyong Ahn', June Seek Choi', Jung Yeol Han', Moon Young Kim', So Yeon Park', Hyun Mee Ryu ${ }^{1,2}$, and Min Hyoung Kim*

'Department of Obstetrics and Gynecology, Cheil General Hospital and Women's Healthcare Center, Dankook University College of Medicine, Seoul, Korea

2Laboratory of Medical Genetics, Medical Research Institute, Cheil General Hospital and Women's Healthcare Center, Seoul, Korea

Purpose: Increased maternal age is a major risk factor for chromosomal abnormalities. The maternal age-specific risk of fetal trisomy was theoretically calculated. We investigated the actual frequency of fetal trisomy between 16 and 24 gestational weeks in pregnant women over the age of 34 at delivery.

Materials and Methods: We retrospectively, over a four-year period, reviewed the medical records of women with singleton pregnancies that started their antenatal care before the 10th week of pregnancy. Pregnant women aged 34 to 45 years at the time of delivery were enrolled and divided into groups of one-year intervals. We investigated the frequency of Down syndrome and all trisomies as a function of the maternal age and compared with the theoretical maternal-age-specific risk. Results: Of the 5,858 pregnant women enrolled in the study, the rate of trisomy 21 was $0.29 \%$ (17 cases). The observed frequencies of trisomy 21 in women with maternal ages of 35 years and 40 years were 1:1,116 and 1:141, respectively. The rate of all trisomies was $0.39 \%$ (23 cases). The observed frequencies of all trisomies in women with maternal ages of 35 years and 40 years were 1:372 and 1:56, respectively.

Conclusion: The frequencies of Down syndrome and all trisomies were proportional to the maternal age. However, the observed frequencies of Down syndrome and all trisomies between the 16 and 24 gestational weeks were lower than the theoretical rates.

Key words: Maternal age, Epidemiology, Trisomy.

\section{Introduction}

Chromosomal abnormalities, including trisomy 21, are some of the most pressing fetal health concerns in the minds of pregnant women and medical providers during the antenatal period. It is well known that fetal chromosomal trisomies increase with advanced maternal age [1]. In the 1980s, the risk of mid-trimester trisomy 21 in a 35-years-old woman at delivery was reported to be 1:270. This risk rapidly rises with increasing maternal age over 35 years. The maternal-age-related risk of

Received: 3 August 2015, Revised: 31 October 2015, Accepted: 1 November 2015, Published: 31 December 2015

*Corresponding author: Min Hyoung Kim, M.D., Ph.D.

Department of Obstetrics and Gynecology, Cheil General Hospital and Women's Healthcare Center, Dankook University College of Medicine, 17 Seoae-ro 1-gil, Jung-gu, Seoul 04619, Korea.

Tel: +82-2-2000-7683, Fax: +82-2-2278-4574, E-mail: obdrmhk@naver.com

Conflict of interest: The authors declare that they do not have any conflicts of interest.

(c) This is an open-access article distributed under the terms of the Creative Commons Attribution Non-Commercial License (http://creativecommons.org/licenses/by-nc/4.0/) which permits unrestricted non-commercial use, distribution, and reproduction in any medium, provided the original work is properly cited.

(c) Copyright 2015 by the Korean Society of Medical Genetics 
chromosomal trisomy originates from calculated regressions on the rates of cytogenetic abnormalities diagnosed during the prenatal period [2]. In previous reports, the pregnancy loss rate after amniocentesis was estimated to be 1:200-300 [3,4]. Based on the theoretically calculated risk of chromosomal trisomy, a maternal age of 35 years, at delivery, was used as the cut-off value for offering a genetic amniocentesis, since the benefit of the detection of trisomy was considered enough to outweigh the risk of amniocentesis.

In Korea, there were a few reports about the frequency of chromosomal abnormalities from amniocentesis in women with advanced maternal age $[5,6]$. In these reports, the frequency of chromosomal abnormalities also increased with maternal age. However, they showed that the frequency of chromosomal abnormalities was limited to women that received invasive tests.

In this study, we investigated the observed frequency of fetal trisomies between 16 and 24 gestational weeks in pregnant women aged 34 years or older at the time of delivery.

\section{Materials and Methods}

We retrospectively, from January 2010 to December 2013, reviewed the medical records of women with singleton pregnancies that started their antenatal care before 10 weeks of pregnancy at a single medical center. This study was approved by the ethics committee of Cheil General Hospital and the Women's Health Care Center (CGH-IRB-2015-1).

Pregnant women over the age of 34 years at the time of delivery were enrolled. Of these, women who fulfilled the following three conditions were included in study population: 1) had a fetal nuchal translucency scan at 11-13 weeks of gestational age, 2) had a serum screening test or amniocentesis, and 3) had an ultrasound scan for the identification of fetal structural anomalies at 20-24 weeks of gestational age. Pregnant women that miscarried or underwent chorionic villus sampling (CVS) before 14 weeks of gestation were excluded. We also excluded women that visited after 10 gestational weeks, since our hospital was a referral center of high-risk pregnancy

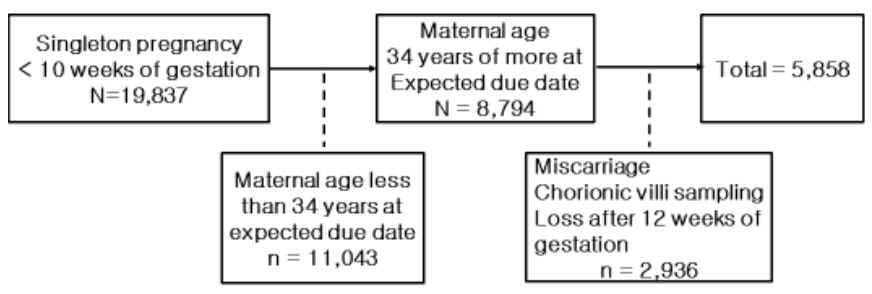

Fig. 1. Enrollment of the study population. and there was a possibility of a bias resulting from those women that had a fetal abnormality detected by ultrasound in another clinic.

We classified the pregnant women aged from 34 to 45 years into groups of one-year intervals. We investigated the frequency of Down syndrome and all trisomies as a function of maternal age between 16 and 24 gestational weeks. The fetuses with a low risk for autosomal trisomy in the nuchal translucency scan on the first trimester ultrasound, the integrated or quad tests, and with a normal structure on the second trimester ultrasound were considered as having a normal karyotype. The fetuses with increased nuchal translucency, but low risk for autosomal trisomy in the integrated or quad tests and identified as a normal structure on the second trimester level II ultrasound, were also considered as having a normal karyotype.

\section{Results}

Women with singleton pregnancies $(19,837)$ were started with antenatal care before 10 weeks of gestation during the study period. Pregnant women younger than 34 years at the expected due date were excluded from the analysis $(n=11,043)$. In addition, 2,936 women were excluded from the analysis due to the following exclusion criteria: miscarriage before 14 weeks of gestation, no first trimester ultrasound for nuchal translucency, subjected to chorionic villi sampling, and loss of follow-up after 12 weeks of gestation. In total, 5,858 pregnant women aged 34 years or older at the time of delivery were included (Fig. 1). The age distribution of the study population is presented in Fig. 2.

In our study population, 17 cases of trisomy 21 were detected, with the ratebeing $0.29 \%$. Of the fetuses with trisomy $21,82.4 \%$ $(n=14)$ were detected in women that selected amniocentesis as the primary test, and $17.6 \%(n=3)$ were detected in women

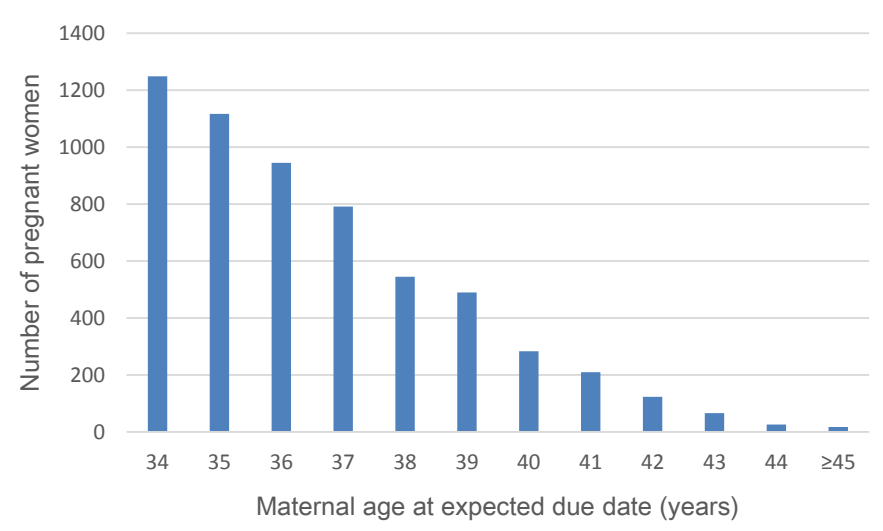

Fig. 2. Age distribution of the study population $(n=5,858)$. 
Table 1. Observed frequency of trisomy 21 and all trisomies between 16 and 24 gestational weeks by maternal age

\begin{tabular}{|cccc|}
\hline $\begin{array}{c}\text { Maternal age at } \\
\text { delivery (yr) }\end{array}$ & Trisomy 21 & All trisomies & Trisomy $21^{\mathrm{a}}$ \\
\hline 34 & $1: 1,248$ & $1: 623$ & $1: 333$ \\
35 & $1: 1,116$ & $1: 372$ & $1: 250$ \\
36 & $1: 314$ & $1: 236$ & $1: 192$ \\
37 & $1: 791$ & $1: 791$ & $1: 149$ \\
38 & $1: 181$ & $1: 181$ & $1: 115$ \\
39 & $1: 162$ & $1: 122$ & $1: 89$ \\
40 & $1: 141$ & $1: 56$ & $1: 69$ \\
41 & $1: 104$ & $1: 104$ & $1: 53$ \\
42 & 0 & $1: 61$ & $1: 41$ \\
43 & 0 & $1: 65$ & $1: 31$ \\
44 & 0 & 0 & $1: 25$ \\
\hline 45 & 0 & 0 & $1: 19$ \\
\hline
\end{tabular}

aTrisomy 21 in the study of Hook et al. (JAMA 1983;249:2034-8) [2].

that selected maternal serum tests as the primary test and that received amniocentesis after counseling due to the high risk for trisomy 21.

The observed frequency of trisomy 21 and all trisomies between 16 and 24 gestational weeks as a function of the maternal age is shown in Table 1. The observed frequencies of trisomy 21 in women with maternal ages of 35 years and 40 years were 1:1,116 and 1:141, respectively. There were 23 cases of all trisomies. The rate of all trisomies at mid-trimester was $0.39 \%$. The observed frequencies of all trisomies in women with maternal ages of 35 years and 40 years were 1:372 and 1:56, respectively.

\section{Discussion}

It is well known that the risk of fetal trisomy, including Down syndrome, increases with maternal age. Traditionally, the cut-off for invasive prenatal test based on advanced maternal age $(\geq 35$ years) originated from the theoretically calculated risk of 1:270 for Down syndrome, at mid-trimester, by Hook et al. [2]. Several studies have reported a similar rate of Down syndrome. In all these reports, the maternal-age-related risk of chromosomal trisomy originates from calculated regressions using the data of prenatally diagnosed cytogenetic abnormalities [7-9].

In this study, we observed Down syndrome and other trisomies between 16 and 24 gestational weeks in pregnant women. The trend of increasing trisomy with increasing maternal age was the same as what has been previously reported $[5,6]$. However, in this study, the observed frequency of Down syndrome as a function of the maternal age was lower than the theoretical rates in previous reports. The risk of Down syndrome for 35-year-old women was 1:1,116, and for 40 year-old women it was 1:141. The risk of all trisomies was 1:372 for 35 year-old women, and for 40 year-old women it was 1:56. In our study, the risk of all trisomies was similar to the calculated risk of Down syndrome found in previous reports. However, our data shows that there is a possibility of a lower observed frequency of fetal trisomy than the calculated frequency of previous reports.

The number of pregnant women with advanced maternal age ( $\geq 35$ years) is increasing. About one third of the women that received antenatal care during our study period had advanced maternal age (data not shown). In 2007, American College of Obstetricians and Gynecologists recommended that a maternal age of 35 years alone should no longer be used as a cutoff in determining who should be offered the invasive testing [10].

Our study has several limitations. First, we excluded cases of Down syndrome and other trisomies detected during the first trimester using CVS. We found 14 cases of trisomies ( 6 cases of Down syndrome, 7 cases of trisomy 18, 1 case of trisomy 13) in women aged $\geq 34$ years using CVS. Unlike the high abortion rate of trisomy 18 and trisomy 13 at the first trimester, Down syndrome is non-lethal and most fetuses with Down syndrome can survive after the first trimester. All of trisomy 21 diagnosed using CVS had an increased nuchal translucency. Two of them had a high possibility of abortion before 16 gestational weeks due to the increased nuchal translucency above $6.0 \mathrm{~mm}$ and severe soft tissue edema. Four of them only had increased nuchal translucency (range, 3.0-4.8 $\mathrm{mm}$ ), giving them the possibility of survival through pregnancy. If these cases are assessed at mid-trimester, the frequency is somewhat increased. Second, we observed the pregnant women until the second trimester ultrasound and did not evaluate the final result of the pregnancy. Thus, there is the possibility of missing cases of Down syndrome that were not prenatally diagnosed.

In conclusion, this study reports the observed frequencies of fetal trisomy between 16 and 24 gestational weeks in all women $\geq 34$ years of age. Although the frequencies of Down syndrome and all trisomies increase with maternal age, the observed frequencies were lower than theoretical risk.

\section{References}

1. Hook EB. Rates of chromosome abnormalities at different maternal ages. Obstet Gynecol 1981;58:282-5.

2. Hook EB, Cross PK, Schreinemachers DM. Chromosomal abnormality 
rates at amniocentesis and in live-born infants. JAMA 1983;249: 2034-8.

3. Chorionic villus sampling and amniocentesis: recommendations for prenatal counseling. Centers for Disease Control and Prevention. MMWR Recomm Rep 1995;44:1-12.

4. Tabor A, Philip J, Madsen M, Bang J, Obel EB, Nørgaard-Pedersen B. Randomised controlled trial of genetic amniocentesis in 4606 lowrisk women. Lancet 1986;1:1287-93.

5. Park IY, Kwon JY, Kim YH, Kim M, Shin JC. Maternal age-specific rates of fetal chromosomal abnormalities at 16-20 weeks' gestation in Korean pregnant women $>$ or $=35$ years of age. Fetal Diagn Ther 2010; 27:214-21.

6. Kim YJ, Lee JE, Kim SH, Shim SS, Cha DH. Maternal age-specific rates of fetal chromosomal abnormalities in Korean pregnant women of advanced maternal age. Obstet Gynecol Sci 2013;56:160-6.

7. Huether CA, Ivanovich J, Goodwin BS, Krivchenia EL, Hertzberg VS, Edmonds LD, et al. Maternal age specific risk rate estimates for Down syndrome among live births in whites and other races from Ohio and metropolitan Atlanta, 1970-1989. J Med Genet 1998;35:482-90.

8. Snijders RJ, Sundberg K, Holzgreve W, Henry G, Nicolaides KH. Maternal age- and gestation-specific risk for trisomy 21. Ultrasound Obstet Gynecol 1999;13:167-70.

9. Morris JK, Mutton DE, Alberman E. Revised estimates of the maternal age specific live birth prevalence of Down's syndrome. J Med Screen 2002;9:2-6.

10. ACOG Committee on Practice Bulletins. ACOG Practice Bulletin No. 77: screening for fetal chromosomal abnormalities. Obstet Gynecol 2007;109:217-27. 